Research Article

\title{
Relating graph energy and Sombor index*
}

\author{
Alper Ülker ${ }^{1}$, Arif Gürsoy ${ }^{2}$, Necla Kırcalı Gürsoy ${ }^{3}$, Ivan Gutman ${ }^{4, \dagger}$ \\ ${ }^{1}$ Department of Mathematics, Ağrı İbrahim Ceçen University, 04100 Ağrl, Turkey \\ ${ }^{2}$ Department of Mathematics, Ege University, 35040 İzmir, Turkey \\ ${ }^{3}$ Tire Kutsan Vocational School, Ege University, 35900 İzmir, Turkey \\ ${ }^{4}$ Faculty of Science, University of Kragujevac, 34000 Kragujevac, Serbia
}

(Received: 29 August 2021. Accepted: 20 September 2021. Published online: 23 September 2021.)

(C) 2021 the authors. This is an open access article under the CC BY (International 4.0) license (www.creativecommons.org/licenses/by/4.0/).

\begin{abstract}
The energy of a graph $(\varepsilon)$ is the sum of absolute values of its eigenvalues, thus it is a graph-spectrum-based quantity. The Sombor index $(S O)$ is a recently conceived vertex-degree-based topological index. We establish various relations between $\varepsilon$ and $S O$, among which are lower and upper bounds. These relations improve and extend earlier results communicated in the paper [A. Ülker, A. Gürsoy, N. K. Gürsoy, MATCH Commun. Math. Comput. Chem. 87 (2022) 51-58].
\end{abstract}

Keywords: energy (of graph); Sombor index; spectrum (of graph); topological index; degree (of vertex).

2020 Mathematics Subject Classification: 05C09, 05C50, 05 C07.

\section{Introduction}

In this paper we are concerned with simple graphs, assumed to be connected. Let $G$ be such a graph, with $n$ vertices, $m$ edges, vertex set $V(G)$, and edge set $E(G)$. The edge connecting the vertices $u$ and $v$ will be denoted by $u v$. The degree (= number of first neighbors) of the vertex $u \in V(G)$ is denoted by $d_{u}$. In addition, $\min _{u \in V(H)} d_{u}=\delta$ and $\max _{u \in V(H)} d_{u}=\Delta$.

Let $\mathbf{A}(G)$ be the $(0,1)$-adjacency matrix of the graph $G$, and let $\lambda_{1}, \lambda_{2}, \ldots, \lambda_{n}$ be its eigenvalues, forming the spectrum of $G$ [3]. The energy of the graph $G$ is defined as

$$
\varepsilon(G)=\sum_{i=1}^{n}\left|\lambda_{i}\right| .
$$

This spectrum-based graph invariant has been extensively studied, both in mathematics [6-8] and in theoretical chemistry [5].

There are nowadays some 50 different bond incident degree (BID) graph invariants of the form

$$
B I D(G)=\sum_{u v \in E(G)} F\left(d_{u}, d_{v}\right)
$$

where $F$ is a suitably chosen function with the property $F(x, y)=F(y, x)$. Among them, a very recently introduced such invariant is the Sombor index, for which $F(x, y)=\sqrt{x^{2}+y^{2}}$. Thus, the Sombor index is defined as [4],

$$
S O(G)=\sum_{u v \in E(G)} \sqrt{d_{u}^{2}+d_{v}^{2}} .
$$

At the first glance, there hardly could be expected that the spectrum-based graph energy $\varepsilon$ and the vertex-degree-based indices $B I D(G)$ are anyhow related. Yet, the first such relation was discovered by Arizmendi and Arizmendi [1], who showed that $\varepsilon(G) \leq 2 R(G)$, where $R(G)$ is the Randić index, $F(x, y)=1 / \sqrt{x y}$. This was followed by Yan et al. [11], who proved that $\varepsilon(G) \geq(2 / \Delta) R(G)$. These results motivated three of the present authors to seek for analogous connections between $\varepsilon$ and $S O$ [10]. They obtained lower and upper bounds between $\varepsilon$ and $S O$ (for details see below). In the present paper we improve and extend these bounds, and offer a few more relations of this kind.

*This paper was finalized on the day when the sad news arrived that Professor Nenad Trinajstić passed away. Let this humble article be dedicated to the memory of the Great Scholar and founder of chemical graph theory.

†Corresponding author (gutman@kg.ac.rs). 


\section{Energy of a vertex and its properties}

In what follows we will much exploit the concept of energy of a vertex, invented by Arizmendi et al. in 2018 [2]. In this section we repeat the basic facts of their theory.

Let the vertices of the graph $G$ be labeled by $v_{1}, v_{2}, \ldots, v_{n}$. Then the energy of the vertex $v_{i}$ is defined as [2]

$$
\varepsilon\left(x_{i}\right)=\left[\left[\mathbf{A}(G) \mathbf{A}(G)^{T}\right]^{1 / 2}\right]_{i i}
$$

where $\mathbf{A}(G)^{T}$ is the transpose of $\mathbf{A}(G)$. It can be shown that [2]

$$
\sum_{i=1}^{n} \varepsilon\left(v_{i}\right)=\varepsilon(G)
$$

Theorem 2.1. [2] Let $G$ be any graph and $v \in V(G)$. Then $\varepsilon(v) \leq \sqrt{d_{v}}$. Equality holds if and only if $v$ is the central vertex of $K_{1, n-1}$.

Proposition 2.1. Let $v$ be the central vertex of the star $K_{1, n-1}$. Then $S O\left(K_{1, n-1}\right)=\varepsilon(v)^{2} \sqrt{\varepsilon(v)^{4}+1}>\varepsilon(v)^{4}$.

Proof. $K_{1, n-1}$ has $m=n-1$ edges, whereas $S O\left(K_{1, n-1}\right)=m \sqrt{m^{2}+1}$. The degree of the central vertex is equal to $m$, which by Theorem 2.1 is equal to $\varepsilon(v)^{2}$.

Theorem 2.2. [2] Let $G$ be a graph with $m \geq 1$ and maximum vertex degree $\Delta$. Then for any $v \in V(G), \varepsilon(v) \geq d_{v} / \Delta$. Equality holds if and only if $G \cong K_{a, a}$ for $a \geq 1$.

\section{First inequality between graph energy and Sombor index}

In [10], the following result was obtained:

Proposition 3.1. [10] Let $G$ be a connected graph with minimum vertex degree $\delta \geq 2$. Then $\varepsilon(G) \leq S O(G)$.

We now strengthen this result as follows:

Theorem 3.1. Let $G$ be a connected graph with $n$ vertices. If $n=2$, then $\varepsilon(G)>S O(G)$. If $n \geq 3$, then $\varepsilon(G)<S O(G)$.

Proof. If $n=2$, then $G \cong K_{2}$. It is easy to compute that $\varepsilon\left(K_{2}\right)=2$ and $S O\left(K_{2}\right)=\sqrt{2}$.

Suppose now that $n \geq 3$. Then the graph $G$ cannot have an edge $u v$ such that $d_{u}=d_{v}=1$. Then the minimal value of the term $\sqrt{d_{u}^{2}+d_{v}^{2}}$ is $\sqrt{5}$, when $d_{u}=1, d_{v}=2$. Therefore,

$$
S O(G) \geq \sqrt{5} m .
$$

Equality in (1) holds if and only if $G \cong K_{1,2}$.

The McClelland inequality is $\varepsilon(G) \leq \sqrt{2 m n}[8,9]$. For connected graphs, equality holds if and only if $G \cong K_{2}$.

Consider first the case that $G$ is a tree. Then, $n=m+1$, and $\sqrt{2 m n}=\sqrt{2 m(m+1)}$. It is now easy to show that $\sqrt{2 m(m+1)}<\sqrt{5} m$ holds for all $m \geq 1$.

If $G$ is a connected cycle-containing graph, then $n \geq m$, and

$$
\sqrt{2 m n} \leq \sqrt{2 m^{2}}=\sqrt{2} m<\sqrt{5} m .
$$

Thus, in all cases,

$$
\sqrt{2 m n}<\sqrt{5} m .
$$

Bearing in mind the inequalities (1) and (2), we have

$$
\varepsilon(G) \leq \sqrt{2 m n}<\sqrt{5} m \leq S O(G) .
$$

We now improve Theorem 3.1, i.e., the inequality $\varepsilon(G)^{2}<S O(G)^{2}$. 
Theorem 3.2. Let $G$ be a connected graph with $n \geq 3$ vertices. If $\delta \geq 2$, then

$$
\varepsilon(G)<\sqrt{\frac{\sqrt{2}}{\delta} S O(G)+n(n-1) \Delta} \quad \text { i.e. } \quad \varepsilon(G)^{2}<\frac{\sqrt{2}}{\delta} S O(G)+n(n-1) \Delta .
$$

If $\delta=1$, then

$$
\varepsilon(G) \leq \sqrt{\frac{2}{\sqrt{5}} S O(G)+n(n-1) \Delta} \quad \text { i.e. } \quad \varepsilon(G)^{2} \leq \frac{2}{\sqrt{5}} S O(G)+n(n-1) \Delta .
$$

Proof. Directly from the definition of Sombor index, he have

$$
\sqrt{2} \delta m \leq S O(G) \leq \sqrt{2} \Delta m .
$$

If $\delta=1$, since $d_{u}=d_{v}$ cannot happen, the left-hand inequality can be improved by $d_{u}=1, d_{v}=2$ as

$$
\sqrt{5} m \leq S O(G)
$$

Thus,

$$
m \leq \begin{cases}\frac{S O(G)}{\sqrt{2} \delta} & \text { if } \delta \geq 2 \\ \frac{S O(G)}{\sqrt{5}} & \text { if } \delta=1 .\end{cases}
$$

Bearing in mind Theorem 2.1,

$$
\begin{aligned}
& \varepsilon(G)=\sum_{v \in V(G)} \varepsilon(v)<\sum_{v \in V(G)} \sqrt{d_{v}}=\sqrt{\left(\sum_{v \in V(G)} \sqrt{d_{v}}\right)^{2}} \\
& =\sqrt{\sum_{v \in V(G)} d_{v}+2 \sum_{\substack{\{u, v\} \subseteq V(G) \\
u \neq v}} \sqrt{d_{u} d_{v}}}=\sqrt{2 m+2 \sum_{\substack{\{u, v\} \subseteq V(G) \\
u \neq v}} \sqrt{d_{u} d_{v}}} \leq \sqrt{2 m+2\left(\begin{array}{c}
n \\
2
\end{array}\right) \Delta}
\end{aligned}
$$

resulting in

$$
\varepsilon(G)<\sqrt{2 m+n(n-1) \Delta} .
$$

Theorem 3.2 follows now by substituting (3) back into (4).

\section{Second inequality between graph energy and Sombor index}

In [10], the following result was obtained:

Proposition 4.1. [10] Let $G$ be a connected graph with maximum vertex degree $\Delta$. Then $\Delta^{3} \varepsilon(G) \geq S O(G)$.

We now establish a stronger upper bound for the Sombor index.

Theorem 4.1. Let $G$ be a connected graph with maximum vertex degree $\Delta$. Then

$$
\frac{\Delta^{2}}{\sqrt{2}} \varepsilon(G) \geq S O(G)
$$

Equality holds if and only if $G \cong K_{a, a}$ for $a \geq 1$.

Proof. By Theorem 2.2 we get

$$
\varepsilon(G)=\sum_{v \in V(G)} \varepsilon(v) \geq \frac{1}{\Delta} \sum_{v \in V(G)} d_{v}
$$

and thus

$$
\varepsilon(G) \geq \frac{2 m}{\Delta}
$$

with equality if and only if $G \cong K_{a, a}$. 
The term $\sqrt{d_{u}^{2}+d_{v}^{2}}$ is maximal if $d_{u}=d_{v}=\Delta$, equal to $\sqrt{2} \Delta$. Then from the definition of Sombor index, it follows that $S O$ is maximal if all vertex degrees are equal to $\Delta$, i.e., if $G$ is a regular graph. Thus

$$
S O(G) \leq \sqrt{2} \Delta m \quad \text { i.e., } \quad m \geq \frac{S O(G)}{\sqrt{2} \Delta}
$$

with equality if and only if $G$ is a regular graph.

Substituting (6) back into (5), we get

$$
\varepsilon(G) \geq \frac{2}{\Delta}\left(\frac{S O(G)}{\sqrt{2} \Delta}\right)
$$

which directly leads to Theorem 4.1.

\section{References}

[1] G. Arizmendi, O. Arizmendi, Energy of a graph and Randić index, Linear Algebra Appl. 609 (2021) 332-338.

[2] O. Arizmendi, J. F. Hidalgo, O. Juarez-Romero, Energy of a vertex, Linear Algebra Appl. 557 (2018) 464-495.

[3] D. Cvetković, P. Rowlinson, S. Simić, An Introduction to the Theory of Graph Spectra, Cambridge Univ. Press, Cambridge, 2010.

[4] I. Gutman, Geometric approach to degree-based topological indices: Sombor indices, MATCH Commun. Math. Comput. Chem. 86 (2021) 11-16.

[5] I. Gutman, B. Furtula, The total $\pi$-electron energy saga, Croat. Chem. Acta 90 (2017) 359-368.

[6] I. Gutman, B. Furtula, Energies of Graphs - Survey, Census, Bibliography, Center Sci. Res., Kragujevac, 2019.

[7] I. Gutman, H. Ramane, Research on graph energies in 2019, MATCH Commun. Math. Comput. Chem. 84 (2020) $277-292$.

[8] X. Li, Y. Shi, I. Gutman, Graph Energy, Springer, New York, 2012.

[9] B. J. McClelland, Properties of the latent roots of a matrix: The estimation of $\pi$-electron energies, J. Chem. Phys. 54 (1971) $640-643$.

[10] A. Ülker, A. Gürsoy, N. K. Gürsoy, The energy and Sombor index of graphs, MATCH Commun. Math. Comput. Chem. 87 (2022) 51-58.

[11] Z. Yan, C. Liu, Y. Pan, J. Li, Energy, Randić index and maximum degree of graphs, MATCH Commun. Math. Comput. Chem. 86 (2021) 539-542. 